\title{
Mixed-Integer Programming Formulation of a Data-Driven Solver in Computational Elasticity
}

\begin{abstract}
Yoshihiro Kanno
This paper presents a mixed-integer quadratic programming formulation of an existing data-driven approach to computational elasticity. This formulation is suitable for application of a standard mixed-integer programming solver, which finds a global optimal solution. Therefore, the results obtained by the presented method can be used as benchmark instances for any other algorithm. Preliminary numerical experiments are performed to compare quality of solutions obtained by the proposed method and a heuristic used in the data-driven computational mechanics.
\end{abstract}

\section{Keywords}

Global optimization; mixed-integer programming; exact solution method; datadriven computing; model-free computational mechanics.

\section{Introduction}

Data-driven computation in elasticity was initiated by Kirchdoerfer and Ortiz [5]. This methodology directly uses the material data set obtained from physical experiments, rather resorting to conventional empirical modeling of a material constitutive law. It attempts to minimize the distance between the data set and the strains and the stresses satisfying the compatibility relation and the force-balance equation. This method has recently been extended to geometrically nonlinear problems [9] and dynamic problems [7]. Also, Kirchdoerfer and Ortiz [6] introduced the information entropy to reduce the variance of the original method in [5].

The method in [5] is regarded as a lazy learning method, where no model is learned from the given data set. In contrast, another data-driven approach proposed by Ibañez et al. [1, 2] is based on the manifold learning, which is one of eager learning methods. Also, to reduce the influence of outliers in a material data set, a method using the local robust regression has been proposed for static analysis of trusses [4].

Attention of this note is focused on a numerical solution of the data-driven approach in [5]. The problem dealt with in [5] is essentially an optimization problem. Algorithm 1 in [5] serves as a heuristic for this optimization problem. Subsequently, pretty much the same heuristics have been used for data-driven computational mechanics [8, 9]. In this note, we show that this optimization problem can be formulated as a mixed-integer quadratic programming (MIQP) problem in a natural manner. This problem can be solved globally with, e.g., a branch-andbound method, because its continuous relaxation is a (convex) quadratic programming problem. Several sophisticated software packages are available for solving MIQP problems. Although the modeling presented in this note is fairly standard in integer optimization, it cannot be found in literature to the best of the author's knowledge.

\footnotetext{
${ }^{\dagger}$ Mathematics and Informatics Center, The University of Tokyo, Hongo 7-3-1, Tokyo 113-8656, Japan. E-mail: kanno@mist.i.u-tokyo.ac.jp.
} 


\section{Problem setting}

In this section, we overview the methodology of data-driven computing in [5]. Although the formulation presented in section 3 can readily be adapted to more general structures, e.g., threedimensional continua, we restrict ourselves to trusses for simple presentation. Throughout the paper, we assume elasticity and small deformation.

Suppose that we are given experimental material data, consisting of pairs of uniaxial strain and uniaxial stress values. We use $D=\left\{\left(\check{\varepsilon}_{1}, \check{\sigma}_{1}\right), \ldots,\left(\check{\varepsilon}_{d}, \breve{\sigma}_{d}\right)\right\}$ to denote the data set, where $\check{\varepsilon}_{j}$ and $\check{\sigma}_{j}$ are the observed strain and stress, respectively, and $d$ is the number of observations.

Consider a truss consisting of this material. Let $m$ and $n$ denote the number of members and the number of degrees of freedom of the nodal displacements, respectively. We use $\varepsilon_{i} \in \mathbb{R}$ and $\boldsymbol{u} \in \mathbb{R}^{n}$ to denote the axial strain of member $i$ and the nodal displacement vector, respectively. The compatibility relations can be described in the form

$$
\varepsilon_{i}=\boldsymbol{b}_{i}^{\top} \boldsymbol{u}, \quad i=1, \ldots, m,
$$

where $\boldsymbol{b}_{i} \in \mathbb{R}^{n}$ is a constant vector.

Let $\sigma_{i} \in \mathbb{R}$ and $\boldsymbol{p} \in \mathbb{R}^{n}$ denote the axial stress of member $i$ and the external load vector, respectively. The force-balance equations are written as

$$
\sum_{i=1}^{m} v_{i} \sigma_{i} \boldsymbol{b}_{i}=\boldsymbol{p}
$$

where $v_{i}$ is the volume of member $i$.

To state the methodology of data-driven computing in [5] succinctly, we attempt to find the points $\left(\varepsilon_{1}, \sigma_{1}\right), \ldots,\left(\varepsilon_{m}, \sigma_{m}\right)$ that are "closest" to $D$, when (1) and (2) are satisfied. Define the distance from point $\left(\varepsilon_{i}, \sigma_{i}\right)$ to data set $D$ by

$$
f\left(\varepsilon_{i}, \sigma_{i}\right)=\min \left\{\sqrt{\frac{v_{i}}{2}}\left\|\left[\begin{array}{c}
\sqrt{c}\left(\varepsilon_{i}-\check{\varepsilon}\right) \\
\left(\sigma_{i}-\check{\sigma}\right) / \sqrt{c}
\end{array}\right]\right\|_{2} \mid(\check{\varepsilon}, \check{\sigma}) \in D\right\},
$$

where $c>0$ is a constant, and $\|\cdot\|_{2}$ denotes the Euclidean norm of a vector. Then we we minimize the sum of squared distances, i.e., $\sum_{i=1}^{m} f\left(\varepsilon_{i}, \sigma_{i}\right)^{2}$.

\section{Mixed-integer quadratic programming formulation}

In this section, we recast the problem described in section 2 as an MIQP problem.

Observe that, in the minimization problem in (3), we select one data point to evaluate the distance from $\left(\varepsilon_{i}, \sigma_{i}\right)$ to $D$. We use $0-1$ variables, $t_{i 1}, \ldots, t_{i d}$, to represent this selection such that $t_{i j}=1$ if $\left(\check{\varepsilon}_{j}, \check{\sigma}_{j}\right)$ is selected, and otherwise $t_{i j}=0$. We see that $\left(e_{i}, s_{i}\right) \in D$ if and only if there 
exist $t_{i 1}, \ldots, t_{i d}$ satisfying

$$
\begin{aligned}
{\left[\begin{array}{l}
e_{i} \\
s_{i}
\end{array}\right] } & =\sum_{j=1}^{d}\left[\begin{array}{l}
\check{\varepsilon}_{j} \\
\check{\sigma}_{j}
\end{array}\right] t_{i j}, \\
\sum_{j=1}^{d} t_{i j} & =1, \\
t_{i j} & \in\{0,1\}, \quad j=1, \ldots, d .
\end{aligned}
$$

From (3), we obtain

$$
f\left(\varepsilon_{i}, \sigma_{i}\right)^{2}=\min _{e_{i}, s_{i}, t_{i 1}, \ldots, t_{i d}}\left\{\frac{1}{2} v_{i} c\left(\varepsilon_{i}-e_{i}\right)^{2}+\frac{1}{2} \frac{v_{i}}{c}\left(\sigma_{i}-s_{i}\right)^{2} \mid(4),(5),(6)\right\} .
$$

It is worth noting that, at the optimal solution of (7), we have $t_{i j}=1$ if $\left(\check{\varepsilon}_{j}, \check{\sigma}_{j}\right)$ is the closest to $\left(\varepsilon_{i}, \sigma_{i}\right)$, and otherwise $t_{i j}=0$.

In the data-driven solver [5], we minimize the sum of $f\left(\varepsilon_{i}, \sigma_{i}\right)^{2}$ in (7) under the constraints in (1) and (2). This problem can be recast as follows:

$$
\begin{array}{ll}
\text { Minimize } & \sum_{i=1}^{m} \frac{1}{2} v_{i} c\left(\varepsilon_{i}-e_{i}\right)^{2}+\sum_{i=1}^{m} \frac{1}{2} \frac{v_{i}}{c}\left(\sigma_{i}-s_{i}\right)^{2} \\
\text { subject to } & {\left[\begin{array}{c}
e_{i} \\
s_{i}
\end{array}\right]=\sum_{j=1}^{d}\left[\begin{array}{c}
\check{\varepsilon}_{j} \\
\check{\sigma}_{j}
\end{array}\right] t_{i j}, \quad i=1, \ldots, m,} \\
& \sum_{j=1}^{d} t_{i j}=1, \quad i=1, \ldots, m, \\
& t_{i j} \in\{0,1\}, \quad i=1, \ldots, m ; j=1, \ldots, d, \\
& \varepsilon_{i}=\boldsymbol{b}_{i}^{\top} \boldsymbol{u}, \quad i=1, \ldots, m, \\
& \sum_{i=1}^{m} v_{i} \sigma_{i} \boldsymbol{b}_{i}=\boldsymbol{p} .
\end{array}
$$

Here, variables to be optimized are $\boldsymbol{u} \in \mathbb{R}^{n}, \varepsilon_{i}, \sigma_{i}, e_{i}, s_{i}$, and $t_{i j}(i=1, \ldots, m ; j=1, \ldots, d)$.

Problem (8) is minimization of a convex quadratic function under some linear equality constraints and some binary constraints. Hence, it is an MIQP problem. This problem can be solved globally with a standard MIP (mixed-integer programming) solver.

Remark 3.1. It is fairly straightforward to extend problem $(8)$ to a continuum discretized by the conventional finite element method. The material data set is now $D=\left\{\left(\check{\boldsymbol{\varepsilon}}_{1}, \check{\boldsymbol{\sigma}}_{1}\right), \ldots,\left(\check{\boldsymbol{\varepsilon}}_{d}, \check{\boldsymbol{\sigma}}_{d}\right)\right\}$, where $\check{\boldsymbol{\varepsilon}}_{j}$ and $\check{\boldsymbol{\sigma}}_{j}$ are second-order symmetric tensors with the dimension three. Instead of the member strain and stress, we attempt to compute the strain and stress tensors at each evaluation point of numerical integration, as well as the nodal displacement vector. The objective function in $8 \mathrm{a}$ is then replaced by

$$
\sum_{i=1}^{m} \frac{1}{2} \rho_{i} \mathbf{C}\left(\boldsymbol{\varepsilon}_{i}-\boldsymbol{e}_{i}\right):\left(\boldsymbol{\varepsilon}_{i}-\boldsymbol{e}_{i}\right)+\sum_{i=1}^{m} \frac{1}{2} \rho_{i} \mathbf{C}^{-1}\left(\boldsymbol{\sigma}_{i}-\boldsymbol{s}_{i}\right):\left(\boldsymbol{\sigma}_{i}-\boldsymbol{s}_{i}\right),
$$




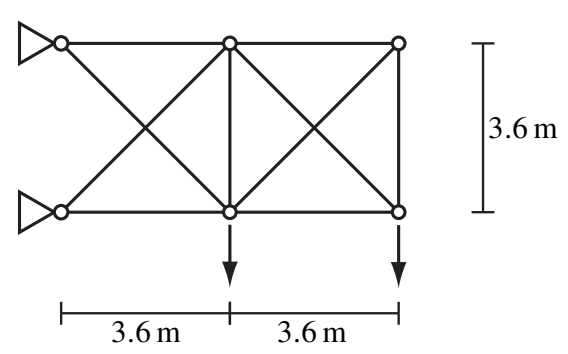

Figure 1: A 10-bar truss.

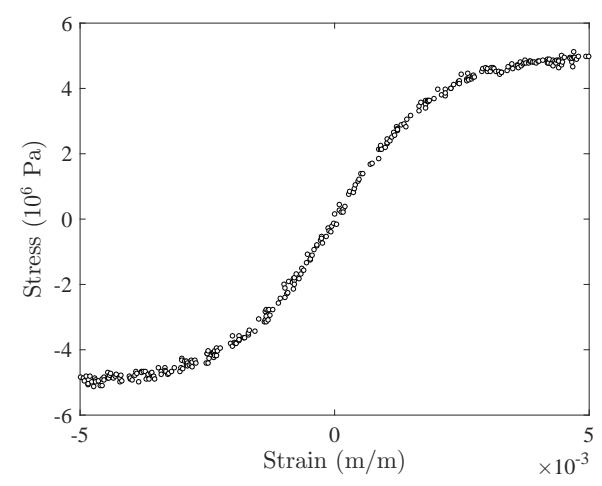

Figure 2: A material data set for the numerical experiments.

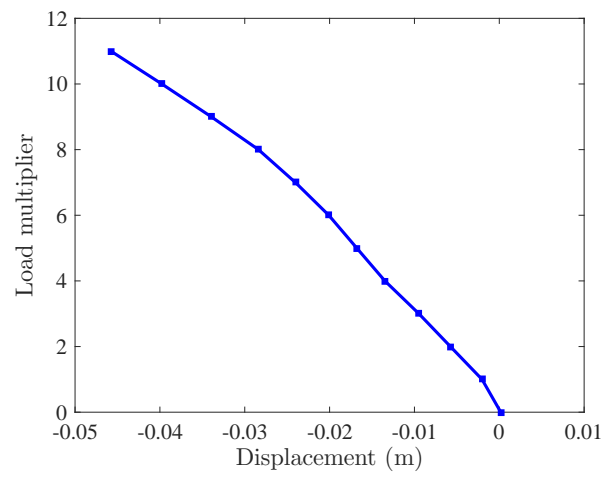

Figure 3: The obtained equilibrium path.

where $\mathbf{C}$ is a constant positive definite fourth-order tensor, and $\rho_{i}>0$ is the weight for the numerical integration. Thus, the objective function is still a convex quadratic function. Also, the constitutive relations in $8 \mathrm{e}$ ) and the force-balance equations in $8 \mathrm{8f}$ remain to be linear equality constraints, because we assume small deformation. Obvious change in $8 \mathrm{~b}$ ) keeps its linearity. Thus, the problem for continua is also an MIQP problem. 


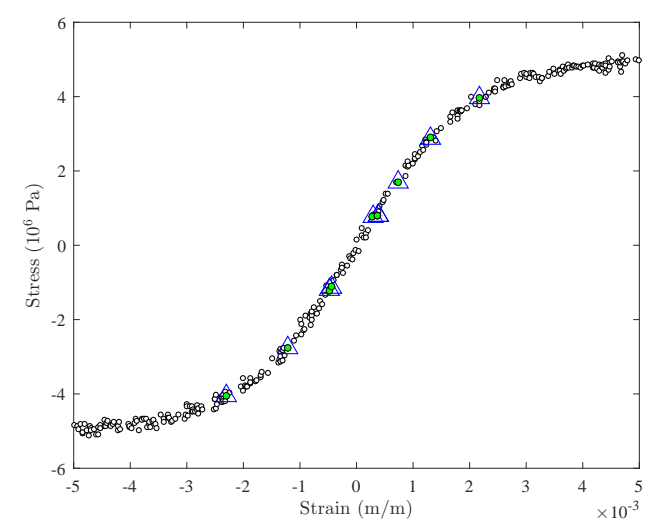

(a)

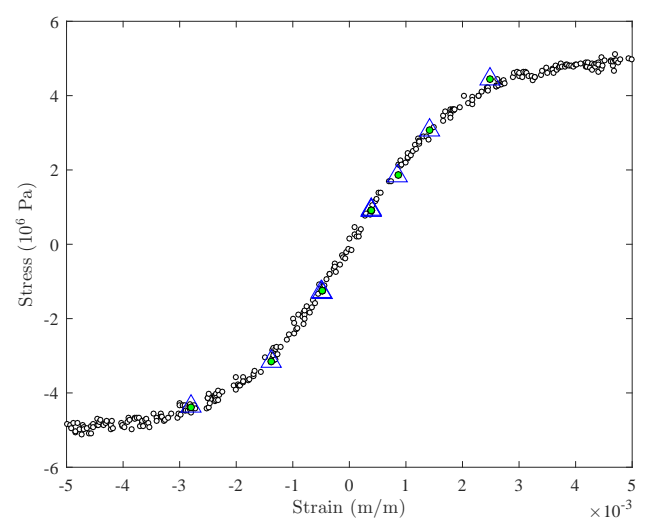

(b)

Figure 4: The solutions obtained by the MIQP approach for (a) $\lambda=10.0$; and (b) $\lambda=11.0$. "triangle" The stress and strain of each member; and "filled circle" the nearest data points.

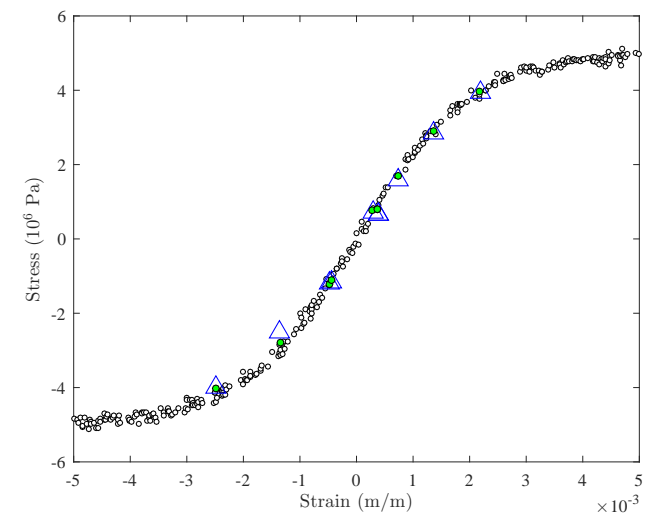

(a)

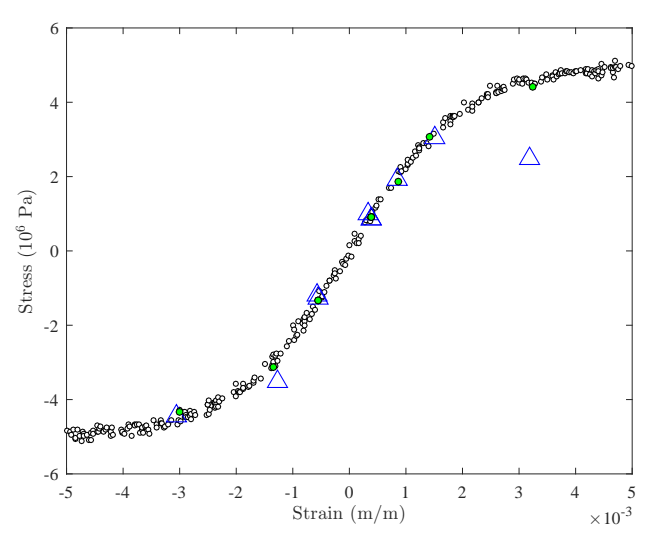

(b)

Figure 5: The solutions obtained by the heuristic in [5] for (a) $\lambda=10.0$; and $[$ b) $\lambda=11.0$. "triangle" The stress and strain of each member; and "filled circle" the nearest data points.

\section{Numerical experiments}

As preliminary numerical experiments, the presented MIQP problem was solved with CPLEX ver. 12.8.0 [3]. Computation was carried out on a $2.2 \mathrm{GHz}$ Intel Core i5-5200 processor with 8 GB RAM. We allowed CPLEX to use up to four threads. The integrality tolerance and the relative MIP gap tolerance (i.e., the tolerance on the relative gap between the objective value of the best feasible solution and that of the best branch-and-bound node remaining) of CPLEX were set to 0, For comparison, Algorithm 1 in [5] was also implemented in Matlab ver. 9.0. We set the initial point for this algorithm to the zero vector.

Consider the planar truss shown in Figure 1. This truss has $m=10$ members and $n=8$ 
degrees of freedom of the nodal displacements. As for the external load, vertical downward forces of $0.4 \lambda$ in $\mathrm{kN}$ are applied at the bottom two nodes as shown in Figure 1 , where $\lambda$ is the load multiplier. Figure 2 shows a material data set, which consists of $d=300$ data points. Hence, our MIQP problem in (8) has $m d=3000$ binary variables. We set constant $c$ in the objective function in (8a) to the mean of $\check{\sigma}_{1} / \check{\varepsilon}_{1}, \ldots, \check{\sigma}_{d} / \check{\varepsilon}_{d}$, which yields $c=1.622 \mathrm{GPa}$.

The proposed method computed the equilibrium path shown in Figure 3 , which depicts the variation of the vertical displacement of the bottom rightmost node with respect to the load multiplier, $\lambda$. Table 1 reports the computational results. For the MIQP approach, "opt." in Table 1 reports the optimal value found by CPLEX, "time" is the computational time, and "\#BnB-node" is the number of enumeration nodes explored by CPLEX. Also, for the heuristic in [5], "obj." is the objective value of the solution found by the method, and "\#iter." is the number of iterations. The computational time required by the MIQP approach is about or less than $60 \mathrm{~s}$. The heuristic [5] does not necessarily converge; namely, for the problem instance with $\lambda=8.0$, it did not converge within 10000 iterations. Also, even if it converges, the obtained solution is not guaranteed to be optimal; indeed, in all the converged cases, its solution has an objective value greater than the one computed by CPLEX.

Figure 4 depicts typical solutions obtained by the proposed method. Here, a triangle indicates a pair of the member strain and stress, $\left(\varepsilon_{i}, \sigma_{i}\right)$, and a filled circle indicates its nearest data point, $\left(e_{i}, s_{i}\right)$. For the same problem instances, Figure 5 shows the solutions obtained by the heuristic [5]. It is worth noting that the objective values of these solutions are more than 100 times larger than the optimal values.

Table 1: Computational results.

\begin{tabular}{rrrrrrr}
\hline & \multicolumn{3}{c}{ MIQP } & & \multicolumn{2}{c}{ Heuristic [5] } \\
\cline { 2 - 3 } \cline { 6 - 7 }$\lambda$ & Opt. $\left(10^{-3} \mathrm{~J}\right)$ & Time $(\mathrm{s})$ & \#BnB-node & & Obj. $\left(10^{-3} \mathrm{~J}\right)$ & \#iter. \\
\hline 0.0 & 6.528 & 39.6 & 10383 & & 1664.071 & 10 \\
1.0 & 23.592 & 29.4 & 9321 & & 321.427 & 17 \\
2.0 & 49.087 & 29.6 & 12417 & & 453.200 & 235 \\
3.0 & 26.810 & 48.6 & 17647 & & 221.352 & 66 \\
4.0 & 41.118 & 87.2 & 74230 & 377.560 & 46 \\
5.0 & 30.580 & 33.4 & 10589 & & 271.801 & 338 \\
6.0 & 13.360 & 33.6 & 21677 & & 490.635 & 27 \\
7.0 & 73.674 & 62.5 & 60549 & 537.069 & 619 \\
8.0 & 106.175 & 43.5 & 31200 & & $-10000)$ \\
9.0 & 26.902 & 36.8 & 26276 & & 395.246 & 25 \\
10.0 & 2.878 & 63.3 & 47830 & & 426.654 & 22 \\
11.0 & 20.120 & 52.5 & 49763 & & 9011.320 & 464 \\
\hline
\end{tabular}




\section{Conclusions}

In this note, we have seen that a problem solved for data-driven computational mechanics [5] can be formulated as a mixed-integer quadratic programming (MIQP) problem. We can solve the MIQP problem globally by using a standard optimization software package. Therefore, the results obtained by the presented approach can be utilized for benchmarking the other algorithms.

Acknowledgments This work is partially supported by JSPS KAKENHI $17 \mathrm{~K} 06633$ and 18K18898.

\section{References}

[1] R. Ibañez, E. Abisset-Chavanne, J. V. Aguado, D. Gonzalez, E. Cueto, F. Chinesta: A manifold learning approach to data-driven computational elasticity and inelasticity. Archives of Computational Methods in Engineering, 25, 47-57 (2018).

[2] R. Ibañez, D. Borzacchiello, J. V. Aguado, E. Abisset-Chavanne, E. Cueto, P. Ladeveze, F. Chinesta: Data-driven non-linear elasticity: Constitutive manifold construction and problem discretization. Computational Mechanics, 60, 813-826 (2017).

[3] IBM ILOG: IBM ILOG CPLEX Optimization Studio Documentation. http://www . ibm. com/support/knowledgecenter/ (Accessed July 2018).

[4] Y. Kanno: Simple heuristic for data-driven computational elasticity with material data involving noise and outliers: A local robust regression approach. Japan Journal of Industrial and Applied Mathematics, to appear. Also arXiv:1708.05794.

[5] T. Kirchdoerfer, M. Ortiz: Data-driven computational mechanics. Computer Methods in Applied Mechanics and Engineering, 304, 81-101 (2016).

[6] T. Kirchdoerfer, M. Ortiz: Data driven computing with noisy material data sets. Computer Methods in Applied Mechanics and Engineering, 326, 622-641 (2017).

[7] T. Kirchdoerfer, M. Ortiz: Data-driven computing in dynamics. International Journal for Numerical Methods in Engineering, 113, 1697-1710 (2018).

[8] A. Leygue, M. Coret, J. Réthoré, L. Stainier, E. Verron: Data-based derivation of material response. Computer Methods in Applied Mechanics and Engineering, 331, 184-196 (2018).

[9] L. T. K. Nguyen, M.-A. Keip: A data-driven approach to nonlinear elasticity. Computers and Structures, 194, 97-115 (2018). 